\title{
Allogenes Stammzellpräparat optimiert die Behandlung komplexer perianaler Fisteln
}

Bis zu $40 \%$ aller Patienten mit Morbus Crohn entwickeln im Krankheitsverlauf Fisteln im Rektumbereich. Speziell bei komplexen Fisteln, vor allem mit begleitender Proktitis, stößt die Chirurgie oft an ihre Grenzen. Hier kann eine zusätzliche Injektion mesenchymaler Stammzellen helfen.

Der größte Risikofaktor für einen fistulierenden Verlauf bei M. Crohn ist eine entzündliche Aktivität des Rektums, erklärte der Chirurg Professor Andreas Pascher, Universität Münster. Während es initial gilt, mit Antibiose oder Abszessdrainage eine Beschwerdebesserung zu erzielen, sind ein Stopp der Fistelsekretion, der Fistelverschluss mit Kontinenzerhalt sowie eine verbesserte Lebensqualität langfristige Ziele.

Bei komplexen perianalen Fisteln hat sich die Applikation mesenchymaler Stammzellen (MSC) als effektive Option erwiesen, berichtete Dr. Roland Scherer, Charité Berlin. Solche MSC aus der Subcutis der Bauchhaut gesunder
Spender werden nach Anzüchtung als standardisiertes Präparat (Darvadstrocel, Alofisel $^{\circledast}$ ) konfektioniert, erläuterte der Chirurg. Die Stammzellsuspension wird dann transanal in Gewebe um die innere Fistelöffnung injiziert. Die immunmodulatorischen Eigenschaften der MSC ermöglichen einen langfristigen Fistelverschluss. In die Zulassungsstudie ADMIRE CD [1] für Darvadstrocel der Phase III wurden 212 M.Crohn-Patienten mit komplexen perianalen sezernierenden Fisteln eingeschlossen, die zuvor nicht auf Antibiotika, Immunmodulatoren oder Anti-TNF-Therapien angesprochen und keine chirurgische Therapie erhalten hatten. Sie erhielten entweder nur eine chirurgische Therapie ( $n=105)$ oder zusätzlich einmalig 120 Mio. MSC injiziert $(n=107)$. Der primäre Endpunkt die kombinierte Remission nach 24 Wochen mit äußerem Verschluss der Fistel ohne Sezernierung bei Fingerdruck und fehlendem MRT-Nachweis von größeren Flüssigkeitsansammlungen - wurde mit Darvadstrocel von $50 \%$, von der Kontrollgruppe von $34 \%$ erreicht (ITT). Zu Woche 104 waren unter MSC noch $56 \%$ und nach rein chirurgischer Behandlung $40 \%$ in klinischer Remission. Darauf basierend erhielt die MSC-Applikation in einem Update der ECCO-Leitlinien [2] den höchsten Evidenzgrad (EL2) der chirurgischen Verfahren bei komplexen perianalen Fisteln zuerkannt, so Scherer abschließend.

\section{Bericht: Dr. Andreas Häckel (hae), Frankfurt am Main}

\section{Literatur}

1. Panés J, et al (2016) ADMIRE CD Study Group Collaborators (2016) Expanded allogeneic adipose-derived mesenchymal stem cells (Cx601) for complex perianal fistulas in Crohn's disease: a phase 3 randomised, double-blind controlled trial. Lancet 388(10051):1281-1290

2. Adamina M, et al (2020) ECCO Guidelines on Therapeutics in Crohn's Disease: Surgical Treatment. J Crohns Colitis 14(2):155-168
Quelle: Virtuelles Satellitensymposium im Rahmen des Deutschen Chirurgenkongresses (DCK) 2021 „Alleine gut, zusammen noch besser: Chirurgie und Gastroenterologie in der Versorgung seltener gastroenterologischer Erkrankungen", 15.04.2021 Veranstalter Takeda

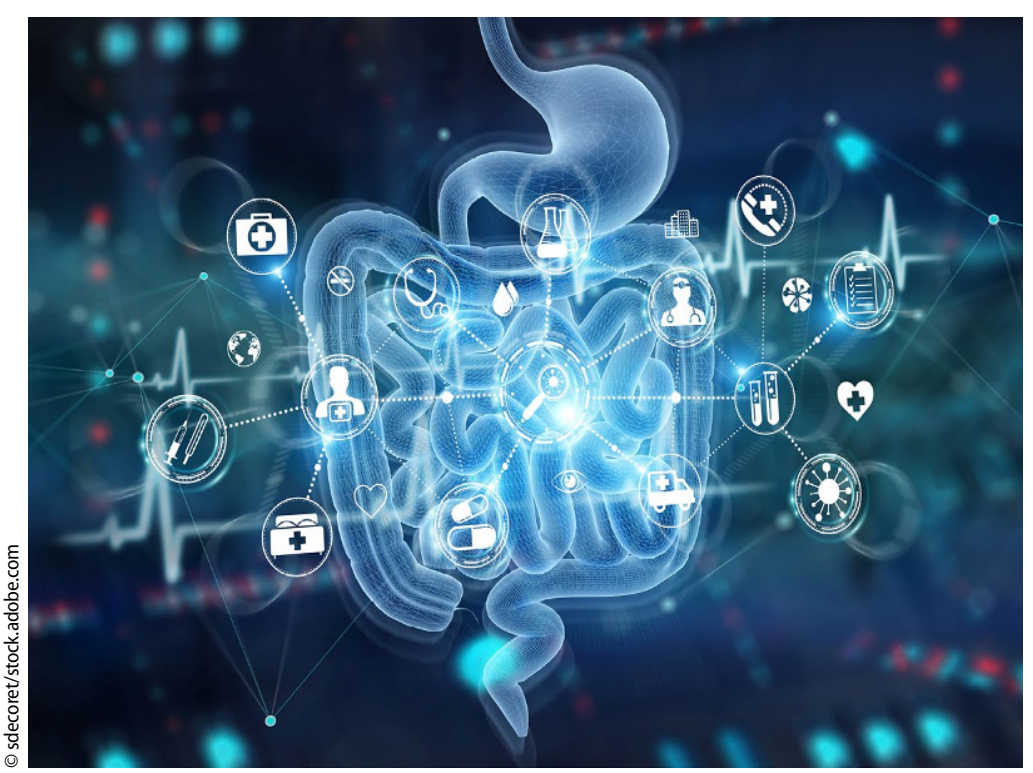

Hinweis des Verlags. Der Verlag bleibt in Hinblick auf geografische Zuordnungen und Gebietsbezeichnungen in veröffentlichten Karten und Institutsadressen neutral.

J. Gastroenterol. Hepatol. Erkr. 2021 · 19:60 https://doi.org/10.1007/s41971-02100098-6

(c) Springer-Verlag GmbH Austria, ein Teil von Springer Nature 2021 